OPEN ACCESS

Edited by:

Moisés Canle,

University of A Coruña, Spain

Reviewed by:

Milan Vraneš,

University of Novi Sad, Serbia

Luis M. Varela,

University of Santiago de Compostela,

Spain

*Correspondence:

Patrik Johansson

patrik.johansson@chalmers.se

tPresent Address: Bodil Ahlström

National Electric Vehicle Sweden $A B$,

Trollhättan, Sweden

Maciej Marczewski,

Faculty of Chemistry, Warsaw University of Technology, Warsaw,

Poland

Specialty section:

This article was submitted to

Green and Sustainable Chemistry,

a section of the journal

Frontiers in Chemistry

Received: 30 September 2018 Accepted: 18 February 2019

Published: 12 March 2019

Citation:

Nilsson-Hallén J, Ahlström B, Marczewski $M$ and Johansson $P$

(2019) Ionic Liquids: A Simple Model to Predict Ion Conductivity Based on DFT Derived Physical Parameters.

Front. Chem. 7:126.

doi: 10.3389/fchem.2019.00126

\section{lonic Liquids: A Simple Model to Predict lon Conductivity Based on DFT Derived Physical Parameters}

\author{
Jonathan Nilsson-Hallén, Bodil Ahlström ${ }^{\dagger}$, Maciej Marczewski ${ }^{\dagger}$ and Patrik Johansson * \\ Department of Physics, Chalmers University of Technology, Gothenburg, Sweden
}

A model able to a priori predict ion conductivities of ionic liquids (ILS) is a desired design tool. We here propose a set of simple conductivity models for ILs composed of small ions by only using data easily derived from standard DFT calculations as input; ion volume, ion mass, ion moment of inertia, and the ion-ion interaction strength. Hence these simple models are totally without any need for experimental parametrization. All model are made from fits of 22 ILs based on 12 different cations and 5 different anions, resulting in correlations vs. experiment of $R^{2} \approx 0.95$ and MAE of $25-36 \%$. Given their (very) simple layout and how fast they can be applied (and re-used), the models allow for ample screening of new IL designs, while not aimed for perfect predictions per se.

Keywords: ionic liquid, conductivity, DFT, model, ion

\section{INTRODUCTION}

Ionic liquids (ILs) represent an interesting class of materials that during the last few decades have been intensely studied (Plechkova and Seddon, 2008; Castner and Wishart, 2010; Thematic issue: Physical chemistry of ionic liquids, 2010; Thematic issue: Ionic liquids, 2012), partly driven by the many potential applications suggested, such as electrolytes in electrochemical energy storage and conversion devices e.g., batteries, fuel cells, solar cells, etc (Armand et al., 2009; Lewandowski and Swiderska-Mocek, 2009; Thematic issue: Ionic liquids for energy applications, 2013). Due to the large number of possible ILs, often estimated to $10^{12}-10^{14}$, or even up to $10^{18}(!)$ (Plechkova and Seddon, 2008), methods for predicting the properties of ILs a priori to any time-consuming synthesis attempts are highly desirable. Any such method needs to take stance at the ion level and should preferably be general, accurate, non-empirical, and fast. Indeed, there are many different standard modeling approaches applied to ILs from ab initio, DFT, MD, to ab initio molecular dynamics (AIMD) (Del Pópolo et al., 2005; Tsuzuki et al., 2005; Borodin, 2009; Kirchner, 2009; Maginn, 2009; Angenendt and Johansson, 2010; Johansson et al., 2010; Ueno et al., 2010; Liu and Maginn, 2012; Tsuzuki, 2012), and more analytical methods (Abbott, 2004, 2005; Matsuda et al., 2007; Slattery et al., 2007; Tochigi and Yamamoto, 2007; Preiss et al., 2010; Eiden et al., 2011). All aim at predicting/estimating melting points (Slattery et al., 2007; Preiss et al., 2010), viscosities (Abbott, 2004; Matsuda et al., 2007; Slattery et al., 2007; Tochigi and Yamamoto, 2007; Ueno et al., 2010; Eiden et al., 2011), and not the least the ionic conductivities (Abbott, 2005; Del Pópolo et al., 2005; Tsuzuki et al., 2005; Matsuda et al., 2007; Slattery et al., 2007; Tochigi and Yamamoto, 2007; Borodin, 2009; Johansson et al., 2010; Ueno et al., 2010; Eiden et al., 2011; Liu and Maginn, 2012; Tsuzuki, 2012). While in general highly successful for their purposes, they often, however, have either limited accuracy, are time-consuming or require ion-specific or empirical parameters. 
From an experimental point of view ion conductivity data are most often provided as Arrhenius plots, $\sigma=\mathrm{f}(1 / \mathrm{T})$, and can for ILs be accurately fitted using the Vogel-Tamman-Fulcher (VTF) equation (Vila et al., 2006),

$$
\sigma=\sigma_{\infty} \exp \left(-\frac{E_{a}}{k_{b}\left(T-T_{0}\right)}\right)
$$

where $\sigma_{\infty}$ is the conductivity at infinite temperature, $\mathrm{E}_{\mathrm{a}}$ is the activation energy, and $\mathrm{T}_{0}$ is a constant related to transformation of the observed glass transition temperature, $\mathrm{T}_{\mathrm{g}}$, to the ideal.

The general features of the VTF equation, together with a lot of other types of expressions, were here used as starting points to generate models for the ionic conductivity of ILs. Many previous IL conductivity models have shown a strong dependency on the ion volumes, but require ion-specific, most often anion-specific, parameters (Tsuzuki et al., 2005; Matsuda et al., 2007; Slattery et al., 2007; Borodin, 2009; Angenendt and Johansson, 2010; Ueno et al., 2010; Tsuzuki, 2012) or resort to analyzing ILs with very similar anions (Johansson et al., 2010). In order to avoid this, which severely limits the generality, we have included not only the ion volumes, but also ion (reduced) masses-as proven to affect the viscosity (Abbott, 2004, 2005; Matsuda et al., 2007), and ion moments of inertia as input parameters to a wide variety of (VTF-based) models. Furthermore, as a combination of (at least) two ions, a cation and an anion, is needed to create any IL, the strength of the specific ion-ion interaction was used as another parameter-more or less as a proxy for the activation energy $\left(\mathrm{E}_{\mathrm{a}}\right)$ in the VTF expression-even if the connection between these is far from obvious (Tsuzuki et al., 2005; Borodin, 2009; Angenendt and Johansson, 2010; Johansson et al., 2010; Ueno et al., 2010; Tsuzuki, 2012). We would also like to stress that we here avoid any additional complexity of IL nano-structuration by only studying ILs with rather short cation alkyl chain lengths. We have also chosen to include imidazolium based ILs with the C2 position methylated as these seem to introduce a specific behavior in terms of ion-ion interaction. All the parameters are obtained starting from standard density functional theory (DFT) calculations on ions and cation-anion ion pairs-and can thus be used to create models totally without any experimental parametrization-one major advantage of the approach chosen.

The various models developed in this study have been fitted and evaluated against in-house measured ionic conductivities. While this clearly limits the statistics of the study in terms of quantity, it improves the quality as it avoids the errors that can result from gathering data from many different sourcesimportant as reported IL conductivities are very sensitive to impurities, not the least water, and the set-up/sample history. From this approach the best fits are reported as largely phenomenological models using some few statistical measures and then discussed in terms of e.g., sensitivity vs. the different input parameters computed and the ILs chosen to study.

\section{MATERIALS AND METHODS}

In total 22 different ILs were used as received from Solvionic with the highest purity available $(99.9$ or $99.5 \%)$. The temperature
TABLE 1 | Name (acronym), volume (V), mass (M), and moment of inertia (I) of ions.

\begin{tabular}{|c|c|c|c|}
\hline Name & $V\left[\AA^{3}\right]$ & $M[u]$ & I $\left[\AA^{2} u\right]$ \\
\hline 1-ethyl-3-methylimidazolium (EMI) & 156 & 111 & 315 \\
\hline 1-butyl-3-methylimidazolium (BMI) & 196 & 139 & 577 \\
\hline 1-hexyl-3-methylimidazolium (HMI) & 246 & 167 & 1,041 \\
\hline 1,3-dimethyl-2-propylimidazolium (PMMI) & 204 & 139 & 536 \\
\hline 1,3-dimethyl-2-butylimidazolium (BMMI) & 224 & 153 & 760 \\
\hline trimethyl-butylammonium ( $\left.\mathrm{Me}_{3} \mathrm{BuN}\right)$ & 111 & 193 & 429 \\
\hline trimethyl-hexylammonium ( $\left.\mathrm{Me}_{3} \mathrm{HexN}\right)$ & 148 & 240 & 934 \\
\hline diethylmethylpropylammonium (Et_2MeProN) & 212 & 130 & 438 \\
\hline 1-propyl-1-methylpyrrolidinium (MPPyr) & 199 & 128 & 409 \\
\hline 1-butyl-1-methylpyrrolidinium (BMPyr) & 222 & 142 & 638 \\
\hline 1-pentyl-1-methylpyrrolidinium (MPePyr) & 242 & 156 & 896 \\
\hline 1-propyl-1-methylpiperidinium (MPPip) & 221 & 142 & 483 \\
\hline tetrafluoroborate $\left(\mathrm{BF}_{4}\right)$ & 71 & 87 & 95 \\
\hline hexafluorophosphate $\left(\mathrm{PF}_{6}\right)$ & 96 & 145 & 201 \\
\hline trifluoromethanesulfonate (Tf) & 115 & 149 & 276 \\
\hline bis(fluorosulfonyl)imide (FSI) & 136 & 180 & 446 \\
\hline bis(trifluoromethanesulfonyl)imide (TFSI) & 199 & 280 & 1,214 \\
\hline
\end{tabular}

dependent ionic conductivity was measured using a Novocontrol broadband dielectric spectrometer in the frequency range $10^{-1}$ $10^{7} \mathrm{~Hz}$. A liquid parallel plate cell, BDS 1308 Novocontrol, with two stainless steel electrodes with a Teflon spacer $(\varnothing=13.2 \mathrm{~mm}$, $\mathrm{d}=0.99 \mathrm{~mm})$ was assembled in an Ar glove box $\left(\mathrm{H}_{2} \mathrm{O}\right.$ and $\mathrm{O}_{2}$ $<1 \mathrm{ppm}$ ) and loaded into a cryo-furnace with $\mathrm{N}_{2}$ gas flow. Data were collected during heating from 298.15 to $368.15 \mathrm{~K}$ in steps of $10 \mathrm{~K}$ and stabilization times of $30 \mathrm{~min}$. The DC conductivities were obtained from the low frequency plateau of the conductivity isotherms by plotting the real part of the complex conductivity as a function of frequency.

Computationally low-energy conformers of all cations (12) and anions (5) (Table 1) constituting the totally 22 ILs investigated experimentally were initially generated by the molecular mechanics based scan routine in Spartan $06^{1}$. Amongst the cations we use the most popular families: imidazolium, EMI etc., and pyrrolidinium, MPPyr (Pyr13) etc. From these the geometries and energies were optimized by DFT, first at the B3LYP/6-311+G* level and subsequently further with M062X/6-311+G* (Vosko et al., 1980; Lee et al., 1988; Becke, 1993; Zhao and Truhlar, 2007), and their 2nd derivatives calculated to ensure the geometries to be local minima energy structures. From the DFT results the ion volumes were calculated as averages of 10 runs using a Monte Carlo (MC) integration scheme of a $0.001 \mathrm{e}^{\mathrm{boh}} \mathrm{b}^{3}$ density envelope and 100 integration points/bohr ${ }^{3}$. The moments of inertia of the ions were calculated as the arithmetic mean of the three principal eigenvalues obtained from the routine in the VMD software (Humphrey et al., 1996).

The lowest energy conformers (B3LYP) for each of the ions were used to generate cation-anion ion pairs, with 20 starting

$\overline{{ }^{1} \text { Spartan '06, Wavefunction Inc. }}$ 
TABLE 2 | Cation-anion ion-pair, $E_{i n t}$, and glass transition temperature $\left(T_{g}\right)$ of the ILS.

\begin{tabular}{|c|c|c|}
\hline Cation-Anion & $\begin{array}{c}E_{\text {int }}(\mathrm{M} 06-2 X / 6- \\
\left.311+G^{*}\right)\left[\mathrm{kJmol}^{-1}\right]\end{array}$ & $T_{g}[K]$ \\
\hline EMl-Tf & -378.9 & - \\
\hline EMI-FSI & -362.3 & - \\
\hline EMI-TFSI & -360.5 & 181 (Fredlake et al., 2004) \\
\hline 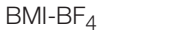 & -385.0 & 188 (Fredlake et al., 2004) \\
\hline $\mathrm{BMI}_{-} \mathrm{PF}_{6}$ & -363.6 & 197 (Fredlake et al., 2004) \\
\hline BMl-Tf & -376.5 & 191 (Tokuda et al., 2006) \\
\hline BMl-TFSI & -367.3 & 187 (Fredlake et al., 2004) \\
\hline $\mathrm{HMI}^{-\mathrm{PF}_{6}}$ & -362.2 & 195 (Harris et al., 2007) \\
\hline HMl-Tf & -374.6 & 189 (Gómez et al., 2013) \\
\hline HMI-TFSI & -363.7 & 189 (Gómez et al., 2013) \\
\hline PMMI-TFSI & -364.2 & - \\
\hline BMMI-TFSI & -363.7 & - \\
\hline $\mathrm{Me}_{3} \mathrm{BuN}-\mathrm{TFSI}$ & -343.9 & 199 (Tokuda et al., 2006) \\
\hline $\mathrm{Me}_{3} \mathrm{HexN}-\mathrm{TFSI}$ & -353.5 & 202 (Taggougui et al., 2008) \\
\hline $\mathrm{Et}_{2} \mathrm{MeProN}-\mathrm{FSI}$ & -340.7 & - \\
\hline MPPyr-FSI & -343.3 & 190 (Zhou et al., 2008) \\
\hline MPPyr-TFSI & -346.8 & 188 (Calvar et al., 2013) \\
\hline BMPyr-Tf & -361.5 & 197 (Calvar et al., 2013) \\
\hline BMPyr-TFSI & -351.2 & 191 (Calvar et al., 2013) \\
\hline MPePyr-TFSI & -352.1 & - \\
\hline MPPip-FSI & -332.9 & - \\
\hline MPPip-TFSI & -338.5 & 192 (Paduszynski et al., 2011) \\
\hline
\end{tabular}

configurations for each pair generated by our in-house developed software SECIL (Angenendt and Johansson). All structures were optimized in two steps; i) B3LYP/6-311+G* - providing a selection of 10 structures/ion pair based on energy and transferred to ii) M06-2X/6-311+ $\mathrm{G}^{*}$. Not all SECIL generated configurations reach SCF convergence, but most B3LYP minima produce M06-2X minima, as verified by the 2nd derivatives. All DFT and MC calculations were made using the Gaussian 03 and 09 program packages (Frisch et al., 2004, 2010). From the M06-2X/6-311+G* data the ion-ion interaction energy, $E_{\text {int }}$ $=\mathrm{E}_{\text {(ion pair })}-\Sigma\left(\mathrm{E}_{\text {cation }}+\mathrm{E}_{\text {anion }}\right)$, was calculated-as a measure of specific ion-ion interaction strength (Table 2). Due to the main aim of arriving at a proof-of-concept method, and not the most performant, and the many other model omissions and simplifications, no comparisons to other DFT functionals (or higher $a b$ initio methods) nor any basis set size effect investigations or basis set super-position error corrections were made.

Using the ion volume (V), mass (M), moment of inertia (I) and ion pair ( $\mathrm{E}_{\text {int }}$, reduced mass) data generated, potential models for the conductivity as function of temperature were formulated and tested with OriginPro $2015^{2}$, using the Levenberg-Marquardt iteration algorithm to fit the data to a predefined function. The most promising models were further combined to achieve better fits, while kept physically sound.

\footnotetext{
${ }^{2}$ OriginLab, Northampton, MA, USA
}

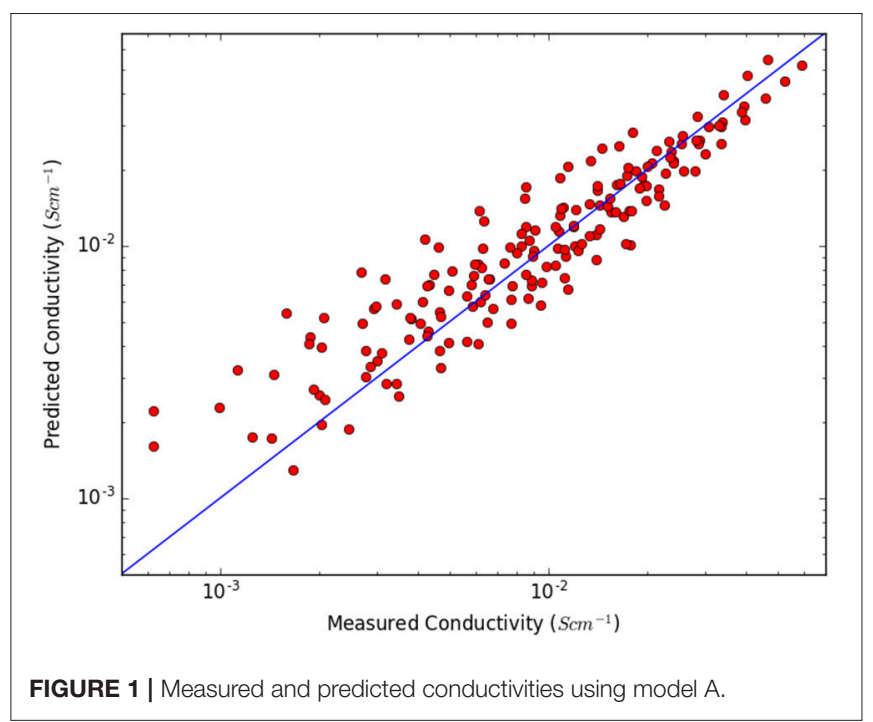

\section{RESULTS AND DISCUSSION}

The computational data generated span an appreciable range of IL ion volumes (71-246 $\left.\AA^{3}\right)$, masses (87-280 u), moments of inertia (95-1214 $\AA^{2} \mathrm{u}$ ), and interaction energies ( -333 to -385 $\left.\mathrm{kJmol}^{-1}\right)$. The first model found with an appreciable fit to the conductivity data, 176 data points for the 22 ILs, is A (Figure 1):

$$
\sigma=\frac{b}{V_{t o t}^{2} I_{+}} \exp \left(c \frac{\text { Eint }}{T-T_{0}}\right)
$$

where the input parameters $V_{\text {tot }}$ is the sum of the cation and anion volumes, $I_{+}$is the moment of inertia of the cation, and $E_{i n t}$ is the interaction energy, while $b, c$, and $T_{0}$ are fitting parameters. That the conductivity is inversely proportional to the ion volumes, as in $\mathbf{A}$, can easily be understood as larger ions both move slower and hinder movement of other ions and this has indeed been found previously (Slattery et al., 2007; Johansson et al., 2010; Tsuzuki, 2012), but why it should be proportional to the inverse of the volume squared is unclear.

It has previously been shown that the viscosity of ILs is affected by the IL ion masses (Abbott, 2004, 2005; Matsuda et al., 2007) and as the conductivity is related to the fluidity, the inverse of the viscosity through the fractional Walden rule, it is reasonable to assume that these could affect the conductivity. The reduced masses, $M_{\text {red }}$, are indeed almost linearly proportional to the volumes $\mathrm{V}_{\text {tot }}$ (Figure 2).

why a second model, A2, with one of the terms exchanged, was investigated-with almost equally good fit (Figure 3):

$$
\sigma=\frac{b}{V_{t o t} M_{r e d} I_{+}} \exp \left(c \frac{\text { Eint }}{T-T_{0}}\right)
$$

In both models $\mathbf{A}$ and $\mathbf{A} 2 \mathrm{~T}_{0}$ has to some extent been treated as a normal fitting parameter. This enables a true prediction of conductivity without any prior IL synthesis, but given the coupling of $\mathrm{T}_{0}$ to $\mathrm{T}_{\mathrm{g}}$, experimental $\mathrm{T}_{\mathrm{g}}$ data as input might 


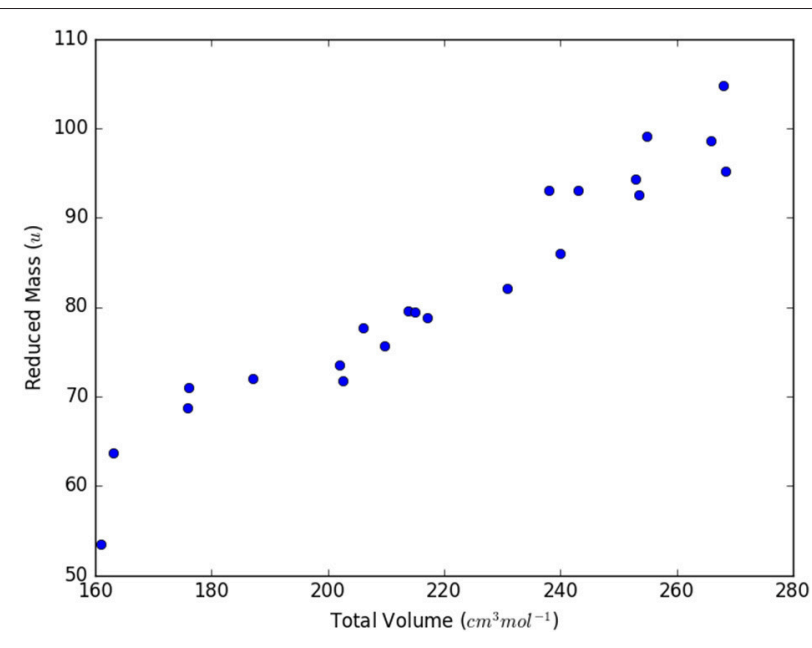

FIGURE 2 | Correlation between total volume and reduced mass for the IL ion pairs.

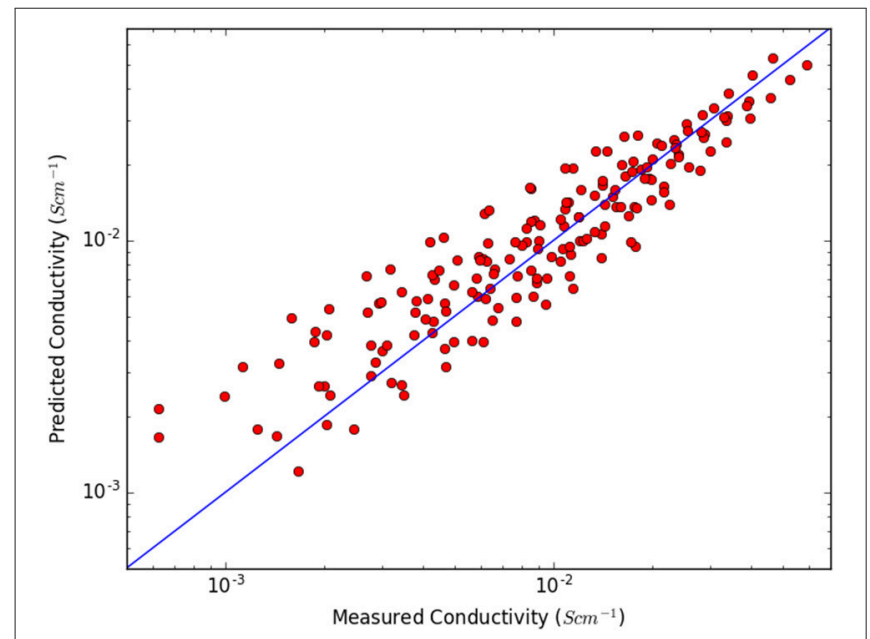

FIGURE 3 | Measured and predicted conductivities using model A2.

improve the fits and models. In the literature $\mathrm{T}_{\mathrm{g}}$ for 15 of the 22 ILs used were found (Table 2) covering the range 181$202 \mathrm{~K}$ (which arguably is a rather limited span in temperature). Applying these data to $\mathbf{A}$ and $\mathbf{A} 2$ result in models $\mathbf{B}$ and B2, respectively:

$$
\begin{array}{r}
\sigma=\frac{b}{V_{\text {tot }}^{2} I_{+}} \exp \left(c \frac{\text { Eint }}{T-\left(T_{g}+d\right)}\right) \\
\sigma=\frac{b}{V_{\text {tot }} M_{\text {red }} I_{+}} \exp \left(c \frac{\text { Eint }}{T-\left(T_{g}+d\right)}\right)
\end{array}
$$

where $d$ is an additional fitting parameter and indeed the fits are slightly improved. Using the reduced data set (15/22) in A and A2 did not improve their fits and thus the improvement indeed comes from the inclusion of $\mathrm{T}_{\mathrm{g}}$ (keeping in mind the limited $\mathrm{T}$ range). All models with their parameter values and statistical measures are summarized in Table 3.
TABLE 3 | Fitting parameter values, correlation coefficients $\left(R^{2}\right)$, and mean absolute errors (MAE) for the four IL conductivity models.

\begin{tabular}{lccccc}
\hline Model & $\mathbf{b}\left[\mathbf{S c m}^{-\mathbf{1}} \AA^{\mathbf{8}} \mathbf{u}\right]$ & $\mathbf{c}\left[\mathbf{K k J}^{\mathbf{- 1}} \mathbf{c}\right.$ & $\mathbf{T}_{\mathbf{0}} / \mathbf{d}[\mathbf{K}]$ & $\boldsymbol{R}^{\mathbf{2}}$ & MAE [\%] \\
\hline A & $1.29 \pm 0.45 \times 10^{7}$ & $-1.01 \pm 0.26$ & $204 \pm 18.0$ & 0.9538 & 35.3 \\
A2 & $2.52 \pm 0.88 \times 10^{6}$ & $-0.90 \pm 0.25$ & $211 \pm 18.0$ & 0.9508 & 36.5 \\
B & $1.09 \pm 0.38 \times 10^{7}$ & $-0.82 \pm 0.23$ & $34.6 \pm 15.9$ & 0.9627 & 24.8 \\
B2 & $3.19 \pm 1.22 \times 10^{6}$ & $-1.01 \pm 0.28$ & $22.6 \pm 17.6$ & 0.9600 & 26.1 \\
\hline
\end{tabular}

\section{CONCLUDING REMARKS}

Two VFT based models for IL conductivity without any experimental input have been developed using volume, mass, and moment of inertia of the constituting ions and the energy of the interaction between cation and anion. In contrast to earlier models there are no anion-specific parameters needed, and in addition moments of inertia of the cations are for the first time included. For the latter feature we initially believed a larger principal moment of inertia to be likely to have a decisive influence due to the elongated shape of many of the ions. However, no such behavior was found in the fits why the arithmetic mean of the three principal values is used. The main influence by the cation moment of inertia is due to many anions being close to spherical. All models without $I_{+}$ performed much worse than the fits here obtained and the inclusion of experimental glass transition temperatures improves the fit, but not to any large extent. From a physical point of view, the ion mass may influence the conductivity, why both $\mathrm{A} 2$ and $\mathrm{B} 2$, replacing one of the volume terms with the reduced masses of the ion pairs, could improve the fit if applied to a larger and more diverge data set (even if they here actually reduce the goodness of the fit). As VFT models in general captures ion conductivities of ILs (and other systems) quite well all of the above in terms of overall agreement come as no major surprise. Phenomenonlogically all the four (VFT) models show the expected behavior; as the volumes, moments of inertia, and masses of the ions increase, the conductivity will approach zero (0), and if they decrease the conductivity will increase. Likewise, if the ion-ion interaction energy increases to infinity the conductivity will approach zero and the conductivity increases with temperature. The inclusion of experimental glass transition temperatures did improve the fit, but there is a rather narrow temperature range for the $\mathrm{T}_{\mathrm{g}}$ used. We stress that we do refrain from trying to connect the input parameters to any direct physical interpretation-such as why the prefactor in the VFT equations seem to relate to the ion volume squared, and how in the exponential the ion-ion interaction energy relate to the activation energy, etc. We do, however, believe it to be straightforward to produce input to these models for any IL of choice-remembering our deliberatively set limits of no nanostructured ILs-for which this model is likely to fail-dependent on how the ion mobility is affected. The DFT calculations will be the time-determining step, even if most data required only needs to be calculated once for each ion, the interaction energy of each ion pair must be obtained separately for each IL. 


\section{AUTHOR CONTRIBUTIONS}

$\mathrm{JN}-\mathrm{H}$ performed the fits based on input from PJ and BA and made a first draft of the paper. MM measured all experimental data. PJ outlined the idea and wrote the final manuscript.

\section{REFERENCES}

Abbott, A. P. (2004). Application of hole theory to the viscosity of ionic and molecular liquids. Chem. Phys. Chem 5, 1242-1246. doi: 10.1002/cphc.200400190

Abbott, A. P. (2005). Model for the conductivity of ionic liquids based on an infinite dilution of holes. Chem. Phys. Chem. 6, 2502-2505. doi: $10.1002 /$ cphc. 200500283

Angenendt, K., and Johansson, P. SECIL- Software Engine for Configuration of Ionic Liquids.

Angenendt, K., and Johansson, P. (2010). Ionic liquid structures from large density functional theory calculations using mindless. Configurations J. Phys. Chem. C 114, 20577-20582. doi: 10.1021/jp104961r

Armand, M., Endres, F., MacFarlane, D. R., Ohno, H., and Scrosati, B. (2009). Ionic-liquid materials for the electrochemical challenges of the future. Nat. Mater. 8, 621. doi: 10.1038/nmat 2448

Becke, A. D. (1993). Density-functional thermochemistry. III. The role of exact exchange. J. Chem. Phys. 98, 5648.

Borodin, O. (2009). Relation between heat of vaporization, ion transport, molar volume, and cation-anion binding energy for ionic liquids. J. Phys. Chem. B 113, 12353-12357. doi: 10.1021/jp9070357

Calvar, N., Gómez, E., Macedo, E. A., and Domínguez, Á. (2013). Thermal analysis and heat capacities of pyridinium and imidazolium ionic liquids. Thermochim. Acta 565, 178-182. doi: 10.1016/j.tca.2013. 05.007

Castner, E. W. Jr. and Wishart, J. F. (2010). Spotlight on ionic liquids. J. Chem. Phys. 132, 120901. doi: 10.1063/1.3373178

Del Pópolo, M. G., Lynden-Bell, R. M., and Kohanoff, J. (2005). Ab Initio molecular dynamics simulation of a room temperature ionic liquid. J. Phys. Chem. B 109, 5895-5902. doi: 10.1021/jp044414g

Eiden, P., Bulut, S., Köchner, T., Friedrich, C., Schubert, T., Krossing, I., et al. (2011). In silico predictions of the temperature-dependent viscosities and electrical conductivities of functionalized and non-functionalized ionic liquids. J. Phys. Chem. B 115, 300-309. doi: 10.1021/jp108059x

Fredlake, C. P., Crosthwaite, J. M., Hert, D. G., Aki, S. N. V. K., and Brennecke J, F. (2004). Thermophysical properties of imidazolium-based ionic liquids. J. Chem. Eng. Data 49, 954-964. doi: 10.1021/je034261a

Frisch, M. J., Trucks, G. W., Schlegel, H. B., Scuseria, G. E., Robb, M. A., Cheeseman, J. R., et al. (2004). Gaussian 03 . (Wallingford, CT).

Frisch, M. J., Trucks, G. W., Schlegel, H. B., Scuseria, G. E., Robb, M. A., Cheeseman, J. R., et al. (2010). Gaussian 09. (Wallingford, CT).

Gómez, E., Calvar, N., Domínguez, Á., and Macedo, E. A. (2013). Thermal analysis and heat capacities of 1-Alkyl-3-methylimidazolium ionic liquids with NTf2-, TFO-, and DCA- anions. Ind. Eng. Chem. Res. 52, 2103-2110. doi: 10.1021/ie3012193

Harris, K. R., Kanakubo, M., and Woolf, L. A. (2007). Temperature and pressure dependence of the viscosity of the ionic liquids 1-hexyl-3methylimidazolium hexafluorophosphate and 1-butyl-3-methylimidazolium bis(trifluoromethylsulfonyl)imide. J. Chem. Eng. Data 52, 1080-1085. doi: $10.1021 /$ je700032n

Humphrey, W., Dalke, A., and Schulten, K. (1996). VMD: visual molecular dynamics. J. Mol. Graph. 14, 33-38.

Johansson, P., Fast, L. E., Matic, A., Appetecchi, G. B., and Passerini, S. (2010).The conductivity of pyrrolidinium and sulfonylimide based ionic liquids: A combined experimental and computational study. Power Sources J. 195, 2074-2076. doi: 10.1016/j.jpowsour.2009. 10.029

Kirchner, B. (2009). Ionic liquids from theoretical investigations. Top. Curr. Chem. 290, 213-262. doi: 10.1007/128_2008_36

\section{ACKNOWLEDGMENTS}

This study was initiated within the project The Physics of Ionic Liquids funded by a basic research grant from the Swedish Research Council (VR)—gratefully acknowledged.

Lee, C., Yang, W., and Parr, R. G. (1988). Development of the Colle-Salvetti correlation-energy formula into a functional of the electron density. Phys. Rev. B 37, 785.

Lewandowski, A., and Swiderska-Mocek, A. (2009). Ionic Liquids as electrolytes for li-ion batteries-an overview of electrochemical studies. Power Sources J. 194, 601-609. doi: 10.1016/j.jpowsour.2009.06.089

Liu, H., and Maginn, E. (2012). An MD study of the applicability of the walden rule and the nernst-einstein model for ionic liquids. Chem. Phys. Chem. 13, 1701-1707. doi: 10.1002/cphc.201200016

Maginn, E. J. (2009). Molecular simulation of ionic liquids: current status and future opportunities. J. Phys. Cond. Matt. 21:373101. doi: 10.1088/0953-8984/21/37/373101

Matsuda, H., Yamamoto, H., Kurihara, K., and Tochigi, K. (2007). Prediction of the ionic conductivity and viscosity of ionic liquids by QSPR using descriptors of group contribution type. J. Comp. Aid Chem. 8, 114-127. doi: $10.2751 /$ jcac. 8.114

Paduszynski, K., Chiyen, J., Ramjugernath, D., Letcher, T. M., and Domanska U (2011). Liquid-liquid phase equilibrium of (piperidinium-based ionic liquid+ an alcohol) binary systems and modelling with NRHB and PCP-SAFT. Fluid Phase Equilibria 305, 43-52. doi: 10.1016/j.fluid.2011.03.005

Plechkova, N. V., and Seddon, K. R. (2008). Applications of ionic liquids in the chemical industry. Chem. Soc. Rev. 37, 123. doi: 10.1039/B006677J

Preiss, U., Bulut, S., and Krossing, I. (2010). In silico prediction of the melting points of ionic liquids from thermodynamic considerations: a case study on 67 salts with a melting point range of $337^{\circ}$ C. J. Phys. Chem. B 114, 11133-11140. doi: 10.1021/jp104679m

Slattery, J. M., Daguenet, C., Dyson, P. J., Schubert, T. J. S., and Krossing, I. (2007). How to predict the physical properties of ionic liquids: a volume-based approach. Angew. Chem. 119, 5480-5484. doi: 10.1002/anie.200700941

Taggougui, M., Diaw, M., Carré, B., Willmann, P., and Lemordant, D. (2008). Solvents in salt electrolyte: Benefits and possible use as electrolyte for lithium-ion battery. Electrochim. Acta 53, 5496-5502. doi: $10.1016 /$ j.electacta.2008.03.012

Thematic issue: Ionic liquids (2012). Faraday Discuss. 154.

Thematic issue: Ionic liquids for energy applications (2013). MRS Bull. 38.

Thematic issue: Physical chemistry of ionic liquids (2010). Phys. Chem. Chem. Phys. 12.

Tochigi, K., and Yamamoto, H. (2007). Estimation of ionic conductivity and viscosity of ionic liquids using a QSPR model. J. Phys. Chem. C 111, 15989-15994. doi: 10.1021/jp073839a

Tokuda, H., Tsuzuki, S., Susan, M. A., Hayamizu, K., and Watanabe, M. (2006). How ionic are room-temperature ionic liquids? an indicator of the physicochemical properties. J. Phys. Chem. B 110, 19593-19600. doi: $10.1021 /$ jp064159v

Tsuzuki, S. (2012).Factors controlling the diffusion of ions in ionic liquids. Chem Phys. Chem. 13, 1664-1670. doi: 10.1002/cphc.201100870

Tsuzuki, S., Tokuda, H., Hayamizu, K., and Watanabe, M. (2005). Magnitude and directionality of interaction in ion pairs of ionic liquids: relationship with ionic conductivity. J. Phys. Chem. B 109, 16474-16481. doi: 10.1021/jp0533628

Ueno, K., Tokuda, H., and Watanabe, M. (2010). Ionicity in ionic liquids: correlation with ionic structure and physicochemical properties. Phys. Chem. Chem. Phys 12, 1649-1658. doi: 10.1039/b921462n

Vila, J. Ginés, P., Pico, J. M., Franjo, C., Jiménez, E., Varela, L.M., et al. (2006). Temperature dependence of the electrical conductivity in EMIM based ionic liquids. Evidence of Vogel-Tamman-Fülcher behavior. Fluid Phase Equilibria 242, 141-146. doi: 10.1016/j.fluid.2006.01.022

Vosko, S. H., Wilk, L., and Nusair, M. (1980). Accurate spin-dependent electron liquid correlation energies for local spin density calculations: a critical analysis. Can. J. Phys. 58, 1200. 
Zhao, Y., and Truhlar, D. G. (2007). The M06 suite of density functionals for main group thermochemistry, thermochemical kinetics, noncovalent interactions, excited states, and transition elements: two new functionals and systematic testing of four M06-class functionals and 12 other functionals. Theor. Chem. Acc. 120, 215-241. doi: 10.1007/s00214-0070310-x

Zhou, Q., Henderson, W. A., Appetecchi, G. B., Montanino, M., and Passerini, S. (2008). Physical and electrochemical properties of N-alkyl$\mathrm{N}$-methylpyrrolidinium bis(fluorosulfonyl)imide ionic liquids: PY13FSI and PY14FSI. J. Phys. Chem. B 112, 13577-13580. doi: 10.1021/jp8 $05419 f$
Conflict of Interest Statement: The authors declare that the research was conducted in the absence of any commercial or financial relationships that could be construed as a potential conflict of interest.

Copyright (C) 2019 Nilsson-Hallén, Ahlström, Marczewski and Johansson. This is an open-access article distributed under the terms of the Creative Commons Attribution License (CC BY). The use, distribution or reproduction in other forums is permitted, provided the original author(s) and the copyright owner(s) are credited and that the original publication in this journal is cited, in accordance with accepted academic practice. No use, distribution or reproduction is permitted which does not comply with these terms. 